\title{
Implementación de un sistema multi-táctil en ambientes educativos para promover y facilitar la evaluación del trabajo colaborativo en el aula
}

\author{
Yadira Quiñonez ${ }^{1}$, Gonzalo Luzardo², Roger Granda ${ }^{2}$ \\ yadiraqui@uas.edu.mx, gluzardo@espol.edu.ec, roger.granda@cti.espol.edu.ec \\ ${ }^{1}$ Universidad Autónoma de Sinaloa, Facultad de Informática, Av. Leonismo Internacional S/N y Av. de los \\ Deportes, Ciudad Universitaria, Mazatlán, Sinaloa, México. \\ ${ }^{2}$ Escuela Superior Politécnica del Litoral, ESPOL, Centro de Tecnologías de Información-Facultad de \\ Ingeniería en Electricidad y Computación, Campus Gustavo Galindo Km 30.5 Vía Perimetral, P.O. Box 09- \\ 01-5863, Guayaquil, Ecuador.
}

DOI: 10.17013/risti.17.66-79

\begin{abstract}
Resumen: Este trabajo presenta un estudio que describe el diseño e implementación de un sistema de mesa multi-táctil de bajo costo que fomenta y facilita la evaluación del trabajo colaborativo dentro de un aula de clase. Se llevaron a cabo pruebas de funcionalidad y usabilidad para validar y verificar el sistema. Adicionalmente, se realizaron dos experimentos para conocer cómo se afecta la percepción de profesores y estudiantes al utilizar este tipo de tecnología. Los aspectos de percepción considerados con los profesores fueron: equidad de carga de trabajo; y facilidad para asignar una calificación individual y grupal. Los aspectos considerados en los estudiantes fueron: equidad de carga de trabajo; capacidad de las herramientas para reflejar su aportación real; y conformidad con calificaciones individuales y grupales obtenidas. De acuerdo con los resultados obtenidos, el uso de esta tecnología tiene un impacto positivo en la percepción de los profesores y estudiantes; los profesores reportaron que el sistema facilita la evaluación del trabajo colaborativo realizado por los estudiantes; por otro lado los estudiantes reportan que este tipo de herramientas ayudan a reflejar de manera más real sus aportaciones en los trabajos colaborativos.
\end{abstract}

Palabras-clave: Diseño colaborativo; evaluación de colaboración; sistema de mesa multi-táctil.

\section{Implementation of a multi-touch system in educational environments to encourage and facilitate the assessment of collaborative work in the classroom}

Abstract: This paper presents a study that describes the design and implementation of a multi-touch tabletop system inexpensive that encourages and facilitates the evaluation of collaborative work in a classroom. Functionality and usability tests were performed to validate and verify the system. Additionally, two experiments were conducted to know how the perception of teacher and students is affected 
by using this technology. The aspects considered in teacher were: workload equality; easiness of grading individuals as well as groups. The aspects considered in students were: workload equality; capability of tools to accurately reflect individual contributions; satisfaction with individual and group score. According to the results, the use of this technology has a positive impact on the perception of teachers and students; teachers reported that the system facilitates the evaluation of the collaborative work done by students; on the other hand, students report that such tools help them to accurately reflect their contributions in collaborative work.

Keywords: Collaborative design; collaborative assessment; multi-tabletop system.

\section{Introducción}

El desarrollo de habilidades de trabajo colaborativo es un aspecto importante durante la formación académica de los estudiantes (Gasca-Hurtado, 2015). En un mundo globalizado y competitivo, los empleadores consideran este tipo de habilidades como un requisito fundamental a la hora de contratar profesionales según (Kaplan, 2014; National Association of Colleges and Employers, 2013; Sinmai, 2014). Si se analiza a profundidad, la forma en la que se trabaja dentro de ambientes colaborativos no ha cambiado significativamente a través de los años, más aún, la tecnología no ha logrado desplazar el uso de herramientas tradicionales de trabajo colaborativo. Por ejemplo, estudios demuestran que el papel y lápiz aún continúan siendo ampliamente utilizados a la hora de diseñar de forma colaborativa (Rick, 2011; Martínez, 2012).

La enseñanza basada en actividades de trabajo colaborativo ha sido uno de los aspectos en que se ha enfocado últimamente la educación superior. Hoy en día, los educadores diseñan actividades, como proyectos y tareas grupales dentro y fuera de clase, que fomentan el trabajo colaborativo dentro y fuera de clase (Xambó, 2013).

El diseño de actividades colaborativas impone un reto importante para los profesores, especialmente aquel relacionado a la evaluación. El proceso de evaluación de una actividad colaborativa se vuelve especialmente difícil debido a que el profesor generalmente cuenta con la versión final del trabajo de sus estudiantes. Las herramientas tradicionales utilizadas no ofrecen información de la carga de trabajo invertida por cada estudiante, su aportación ni la calidad de la misma, que le permita al profesor, de forma objetiva, evaluar y asignar una calificación a cada estudiante (Hayes, 2003; Martínez, 2012).

Investigaciones recientes han encontrado que la percepción de una evaluación injusta debe ser tomada en cuenta por los profesores, ya que ésta es predictora de la motivación, el aprendizaje, e incluso la agresividad que los estudiantes pueden demostrar hacia la materia en particular (Chory-Assad, 2002).

Investigaciones previas (Rick, 2011; Martínez, 2011; Martínez, 2012, Martínez 2012) relacionadas al uso de superficies colaborativas en la educación han demostrado el potencial de éstas para asistir en tareas grupales dentro de un aula de clase, así como su efecto positivo que tienen en los estudiantes para promover el trabajo en grupo. A pesar de esto, poca investigación ha explorado el uso de mesas multi-táctiles (MMT) en ambientes reales de aprendizaje según (Martínez, 2012; Martínez, 2012; AlAgha, 2010). Adicionalmente, estudios específicos relacionados al trabajo en grupo en aulas de clase 
se han enfocado principalmente en: ayudar a los profesores a controlar el trabajo grupal y ofrecer visualización de datos referentes a la elaboración de tareas grupales (Martínez, 2011). Sin embargo, se ha prestado poca atención en el efecto en la percepción de los profesores y alumnos que provoca el uso de esta tecnología.

Actualmente, en la literatura existe cuatro clases de sistemas de MMT: mesas digitales (Wellner, 1993), mesas de trabajo (Cutler, 1997), mesas de dibujo (Buxton, 2000), y mesas de colaboración (Stahl, 2002). El presente trabajo de investigación se centra en el diseño e implementación de una mesa de colaboración para la educación. Se describe el diseño e implementación de un sistema multi-táctil de bajo costo utilizando visión por ordenador, el cual permite mejorar el análisis y posterior evaluación del trabajo colaborativo realizado por un grupo de estudiantes. De igual forma, se presenta un caso de estudio con el fin de validar el sistema propuesto.

Este documento está estructurado de la siguiente manera: primero una revisión de literatura, donde se exploran trabajos previos relacionados al uso de mesas multitáctiles en la educación. A continuación, se hace una descripción de los requerimientos que se consideraron para el desarrollo del sistema propuesto y luego una descripción de su implementación. Posteriormente, se describe la metodología utilizada en los experimentos, en donde se explica el contexto de investigación, las pruebas de funcionalidad, de usabilidad, los detalles de los experimentos realizados y los resultados obtenidos. Al final se realiza una discusión de los resultados y se realizan recomendaciones para trabajos futuros.

\section{Trabajos relacionados}

En los últimos años, la comunidad científica ha avanzado en el estudio de la aplicación de nuevas herramientas tecnológicas (Vázquez-Reyes, 2015) que permiten facilitar el envío y control de actividades colaborativas y resolver en parte los inconvenientes antes mencionados. Según (Dillenbourg, 2011; Higgins, 2011) han desarrollado sistemas de superficies multi-táctiles que permiten facilitar y apoyar el aprendizaje colaborativo en el aula. Estos sistemas fomentan el compromiso, la participación y la creatividad entre los estudiantes, de tal forma que los estudiantes puedan conducir su propio aprendizaje. Así mismo, Mercier et al. (Mercier, 2013) desarrollaron un tablero interactivo llamado NumberNet que permite realizar experimentos virtuales de matemáticas. Del mismo modo, Tyng et al. describen el desarrollo de una aplicación visual de matemáticas para estudiantes de edad preescolar llamada MEL-Vis, que se ejecuta en una mesa multitáctil (Tyng, 2011). Ambos trabajos presentan soluciones viables a través del análisis cualitativo y cuantitativo de resultados experimentales.

Por otro lado, en el trabajo de Sinmai et al. presentan un sistema multitáctil llamado MindMap, el cual fomenta la creación de mapas mentales de forma colaborativa (Sinmai, 2014). Para esto, el sistema brinda la posibilidad de que los estudiantes puedan interactuar de manera grupal e independiente. Cada usuario tiene la posibilidad de crear nodos rectangulares para representar sus ideas. Estos son alimentados de información a través de un teclado virtual o una tableta. Cada nodo "idea" puede ser arrastrado a través de toda la superficie y ser conectado directamente a otros nodos no necesariamente creados por el mismo estudiante, fomentando la interacción. Una particularidad de esta 
aplicación es la definición de espacios personales de cada usuario donde se crean los elementos personales y un espacio público para la interacción con otros usuarios. En este trabajo, los autores describen los requerimientos que guiaron el desarrollo de la solución, los cuales fueron: soporte multiusuarios, definición de un espacio personal y la utilización de dispositivos móviles para alimentar información. Al concluir, los autores demostraron que combinar el uso de mesas multi-táctiles y dispositivos móviles fomenta el trabajo en equipo.

Jones et al. proponen una solución llamada TATIN-PIC, el cual consiste en una mesa multi-táctil y una pizarra vertical, la cual permite la generación de lluvias de ideas a través de diagramas colaborativos. Los diagramas son desarrollados a través de la creación de pequeñas notas rectangulares o "post-it", las cuales representan las ideas. Las notas son creadas a través de la realización de gestos sobre la superficie, la información textual es ingresada a través de un teclado virtual o por medio de reconocimiento de voz. El trabajo total resumido en proyectado en la pizarra con el objetivo de favorecer la discusión y la convergencia de las ideas sobre la solución de un diseño colaborativo (Jones, 2012).

Martínez et al. presentan una solución de mesa multi-táctil llamada Collaid, la cual permite la creación de mapas conceptuales de forma colaborativa (Martínez, 2011). Este sistema fue desarrollado con el objetivo de mejorar el aprendizaje de los estudiantes, así como fomentar el trabajo en equipo en el aula de clases. Collaid utiliza un sensor para identificar la posición de cada usuario, con el fin de ayudar a determinar la contribución de cada uno de ellos sobre el trabajo desarrollado. La información es ingresada por los estudiantes a través de un teclado virtual. Esta solución se integra con un componente de supervisión que es utilizado por el profesor para conocer en todo momento el estado de desarrollo del trabajo elaborado por los estudiantes.

Aunque existen varias soluciones propuestas cuyo objetivo es ayudar al trabajo colaborativo, algunos problemas deben ser resueltos aún. Cuestiones tales como: la restricción del tamaño de la superficie de trabajo, el seguimiento y la supervisión del trabajo colaborativo, versatilidad del sistema número de personas que pueden participar, la complejidad de la configuración del entorno de trabajo, el costo de implementación y herramientas disponibles, hacen necesario profundizar la investigación en este campo.

La revisión de la literatura demuestra que la mayoría se enfocan en asistir a tareas colaborativas de tipo genéricas tales como lluvias de ideas, sketching, mapas mentales, entre otras (Frisch, 2010; Wobbrock, 2009; Xambó, 2013). De igual forma se evidencia la falta de versatilidad de dichas soluciones, por cuanto los sistemas no pueden ser utilizados para dar soporte a diferentes aplicaciones colaborativas.

\section{Sistema multi-táctil propuesto}

\subsection{Características de diseño}

A continuación, se describen las características de diseño que fueron definidas para el sistema propuesto:

1. Soporte multiusuarios: al igual que las soluciones revisadas (Jones, 2012; Sinmai, 2014), esta característica es necesaria para poder brindar la capacidad 
de utilizar el sistema varios usuarios al mismo tiempo.

2. Interacción con objetos tangibles: proveer al estudiante de instrumentos que sean más naturales de utilizar ya que conducen a una menor carga cognitiva (Shen, 2006).

3. Libertad de movimiento: a diferencia de otras soluciones revisadas, el sistema debe permitir libre movimiento a los estudiantes. Xambó et al, advierte de los efectos en la creatividad cuando se definen lugares estáticos de trabajo (Xambó, 2013).

4. Soporte al ingreso de información: al igual que TATINPIC, soporta el ingreso de información a través de tabletas (Jones, 2012).

5. Diferenciación de las aportaciones basadas en color: se ha considerado esta característica con el fin de facilitar al profesor la distinción de aportaciones individuales de los alumnos.

6. Monitoreo y almacenamiento de acciones de usuarios: tiene la finalidad de proveerle herramientas al profesor para controlar múltiples grupos de trabajo de manera simultánea. Además, brindar una funcionalidad de almacenamiento del trabajo, para ofrecerle al profesor la facilidad de evaluar los trabajos una vez que las sesiones de tareas hayan terminado.

\subsection{Esquema físico}

Los estudiantes pueden interactuar con el sistema a través de una superficie colaborativa multi-táctil, plumas digitales y tabletas. Por otra parte, los profesores solo necesitan un dispositivo con un navegador Web para acceder al sistema. La interfaz Web del profesor le permite a este monitorear en tiempo real el progreso de los estudiantes durante el desarrollo de su trabajo. La figura 1 muestra una vista del esquema físico del sistema propuesto.

Los estudiantes pueden interactuar con el sistema a través de una superficie colaborativa multitáctil, plumas digitales y tabletas. Por otra parte, los profesores solo necesitan un dispositivo con un navegador Web para acceder al sistema. La interfaz Web del profesor le permite a este monitorear en tiempo real el progreso de los estudiantes durante el desarrollo de su trabajo. La figura 1 muestra una vista del esquema físico del sistema propuesto.

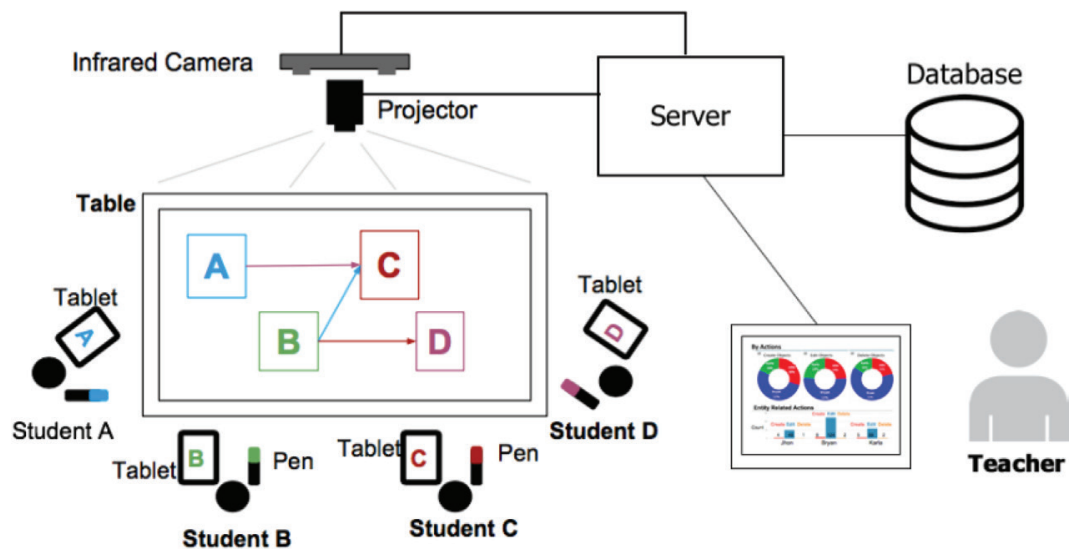

Figura 1 - Esquema físico de la superficie colaborativa 
1. Hardware: La solución está compuesta por un mini proyector (AAXA Technologies P30o Pico Projector) y un Optitrack Motion Tracking V.120 Duo (Figura 2). También se utiliza un computador CoreI5 con 4GB RAM, 500GB HDD, tabletas Samsung Galaxy Tab 3 y plumas con marcadores infrarrojos. El proyector y el Optitrack se encuentran ubicados por encima de una mesa. Una aplicación colaborativa en proyectada en la mesa, en donde los estudiantes interactúan a través de plumas con marcadores infrarrojos y utilizan tabletas para ingresar texto.

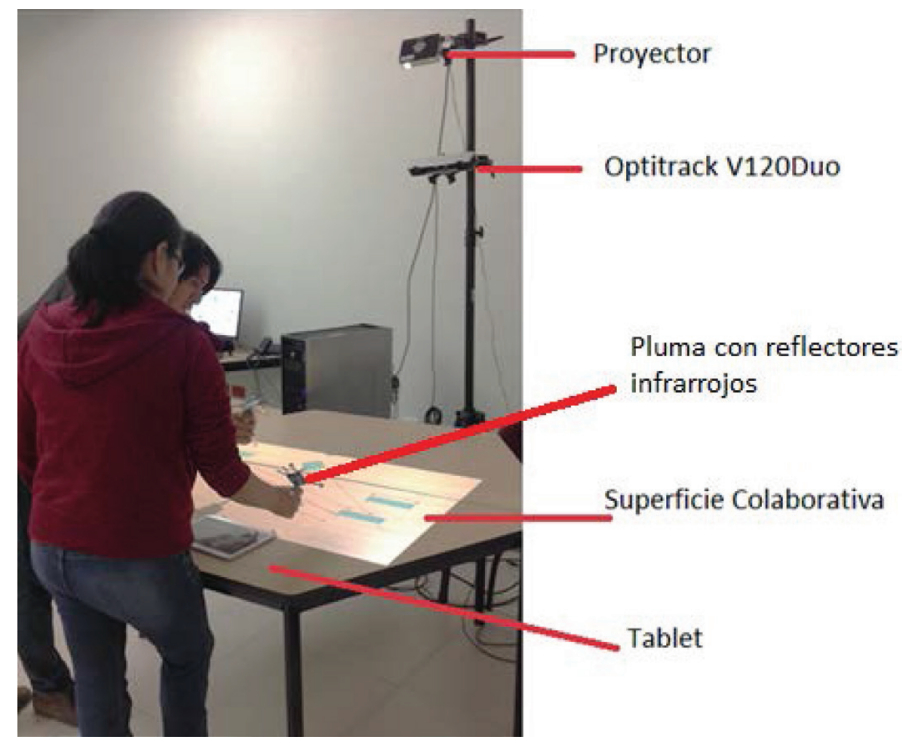

Figura 2 - Implementación física de la superficie colaborativa

2. Software: El sistema está compuesto por: el cliente de control colaborativo, el Server Tracking, un sistema Web para el estudiante y un sistema Web para el profesor. La aplicación Web para el estudiante, le permite iniciar sesión en el sistema y editar texto en la aplicación colaborativa utilizando la tableta. Esta aplicación fue desarrollada en Python utilizando Django Framework. El cliente de control colaborativo es responsable de dos tareas: reconocer trazos utilizando algoritmos de visión por computador y dibujar sobre la interfaz. El cliente fue implementado en el framework Multitouch for Java (MT4J). Cada trazo realizado sobre la superficie es procesado por la librería PaleoSketch (Paulson, 2008.) Esta librería convierte los trazos en formas como rectángulos, círculos y líneas. Los trazos son realizados usando plumas con 3 marcadores infrarrojos. El servidor de rastreo mantiene el seguimiento de las plumas utilizando la librería Camera SDK provisto por Optitrack. Cuando un estudiante dibuja sobre la superficie, un evento de "touch" es generado utilizando el protocolo TUIO (Tangible User interface) y es enviado al cliente de control colaborativo. Las figuras y texto realizados por cada estudiante son dibujados con diferentes colores para diferenciar a cada miembro del grupo. Todas las acciones en la 
superficie son guardadas en un archivo JSON. Cuando los estudiantes finalizan su tarea, pueden descargar este archivo. La figura 3 muestran una vista de los estudiantes interactuando con el sistema.

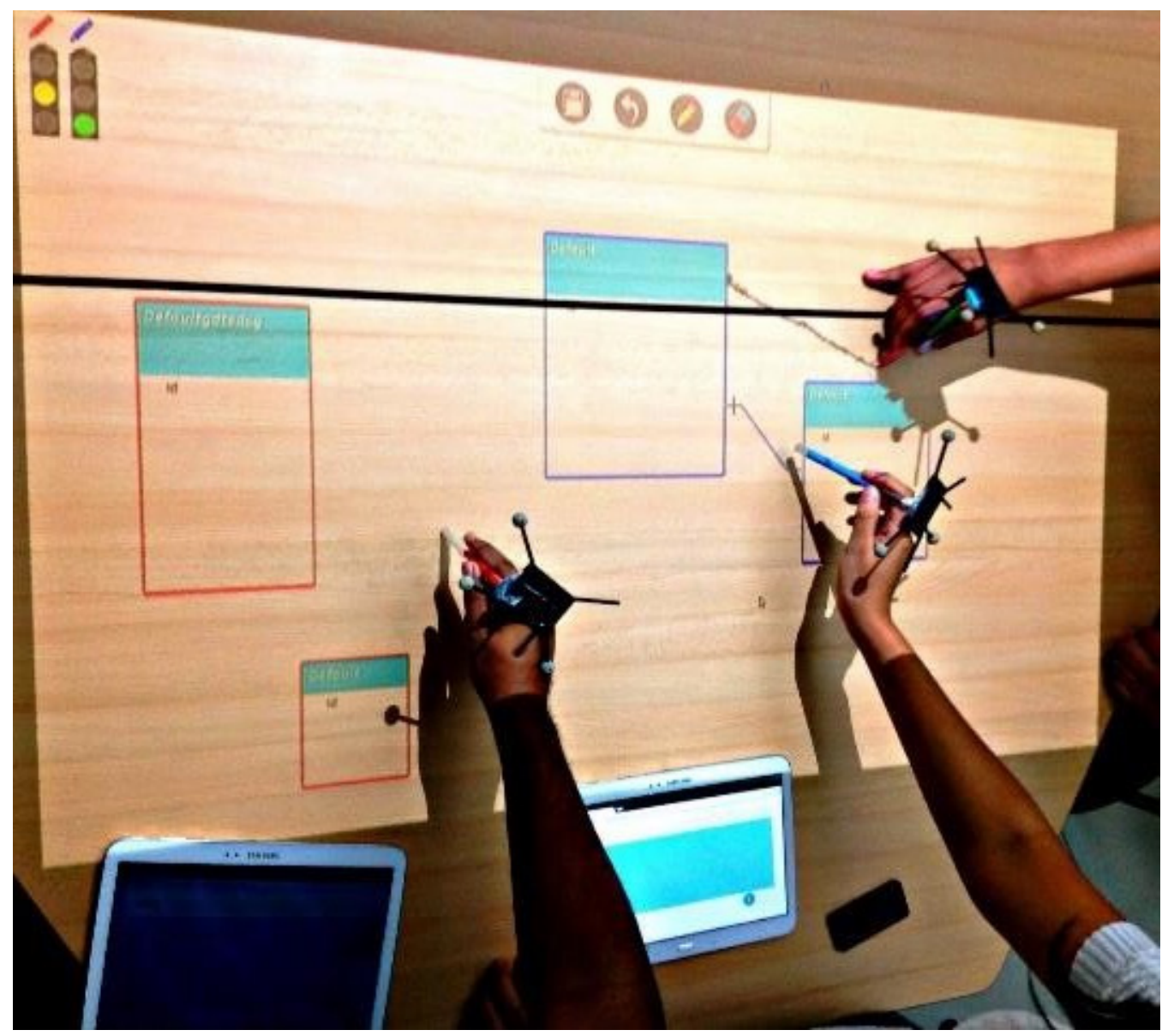

Figura 3 - Tarea grupal de estudiantes utilizando la superficie colaborativa mediante plumas sobre la mesa interactiva

Por otro lado, la interfaz Web del profesor le permite a éste evaluar las actividades de los estudiantes. El sistema mide los niveles de actividad que tiene cada estudiante. En tiempo real, el sistema presenta unas alertas de color que cambian de verde a amarillo o rojo, según el nivel de actividad que haya tenido el estudiante en los últimos 5 minutos (Figura 4a). Verde significa que el estudiante está trabajando activamente, rojo significa que el estudiante ha dejado de hacer acciones en los últimos 5 minutos. El sistema también permite visualizar un resumen de los aportes de cada estudiante al trabajo colaborativo. En este, se muestran los porcentajes de contribución a la tarea, y a un nivel 
más detallado, también se muestra el porcentaje de participación por cada tipo de acción realizada (Figura 4b). Por último, el profesor tiene la capacidad de utilizar el archivo JSON que provee la superficie colaborativa para poder reproducir, en su interfaz Web, todo el proceso de creación de diagrama colaborativo (figura 5).

\subsection{Diseño de la interacción}

La interacción que se propone se basa en objetos tangibles y se propone la utilización de plumas con marcas infrarrojas para dibujar sobre la superficie interactiva. En la figura 6 se muestra un ejemplo de interacción diseñada para el trazado de Entidades en una aplicación de diseño de base de datos. Lo primero que se hace es dibujar un cuadrado que va pintado del color correspondiente a la pluma; una vez reconocido el trazo se dibuja una entidad del mismo color.

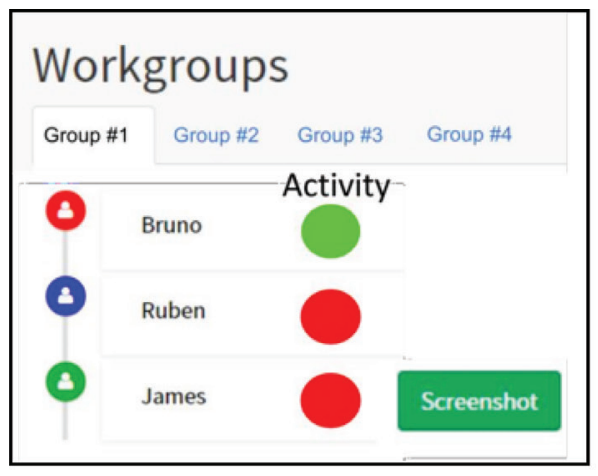

a)

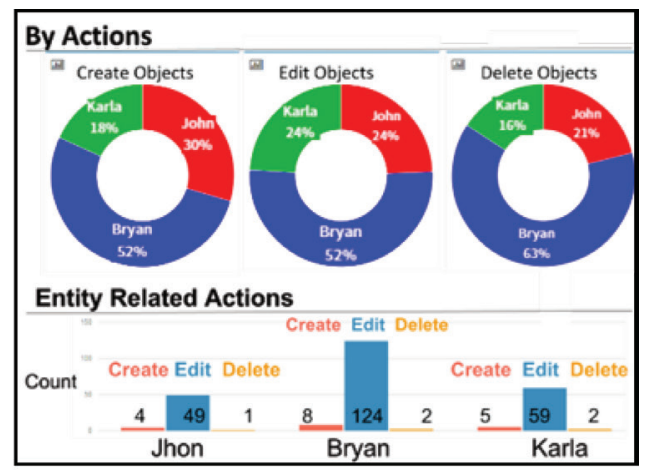

b)

Figura 4 - a) Alertas de colores utilizadas para indicar la actividad de cada estudiante.

b) Porcentajes de participación de los estudiantes

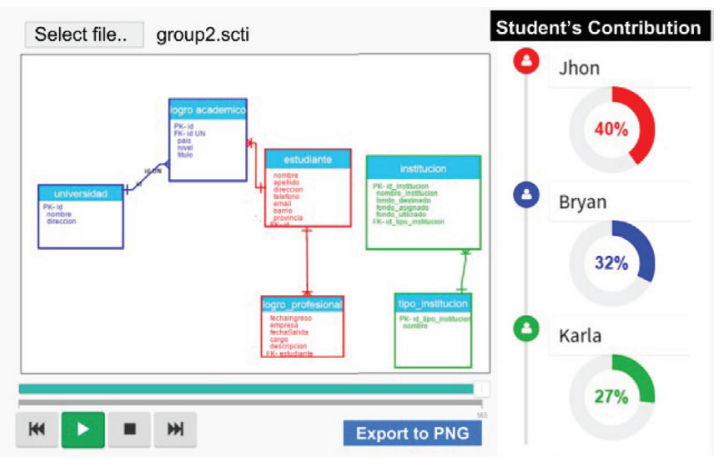

Figura 5 - Reproductor utilizado para observar el proceso de elaboración de la tarea colaborativa 


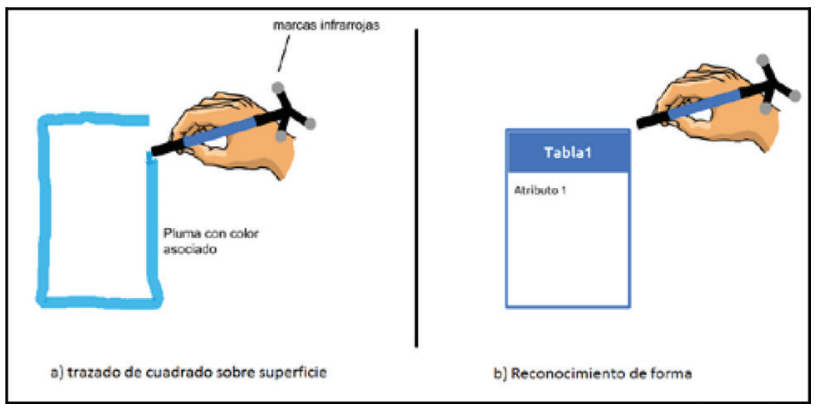

Figura 6 - Interacción con pluma sobre la superficie colaborativa para la creación de entidades

\subsection{Componentes del sistema}

En la figura 7 se muestran los componentes y las interfaces principales que proveen los requerimientos necesarios para el buen funcionamiento del sistema propuesto. En primer lugar, el componente de captura de movimiento o denominado Servidor de rastreo; permite conocer la posición de las plumas en todo momento para determinar la interacción con la superficie proyectada. En segundo lugar, el componente de visualización y control colaborativo o Cliente Gráfico; se encarga de la representación visual de los objetos en la superficie colaborativa, además de ser el encargado del reconocimiento de los trazos realizados por los usuarios. Por último, el componente de autentificación y control individual o aplicación Web; permite a los usuarios autentificarse e ingresar información al trabajo colaborativo, y al mismo tiempo, se utiliza para proveer la interfaz de monitoreo y evaluación del profesor.

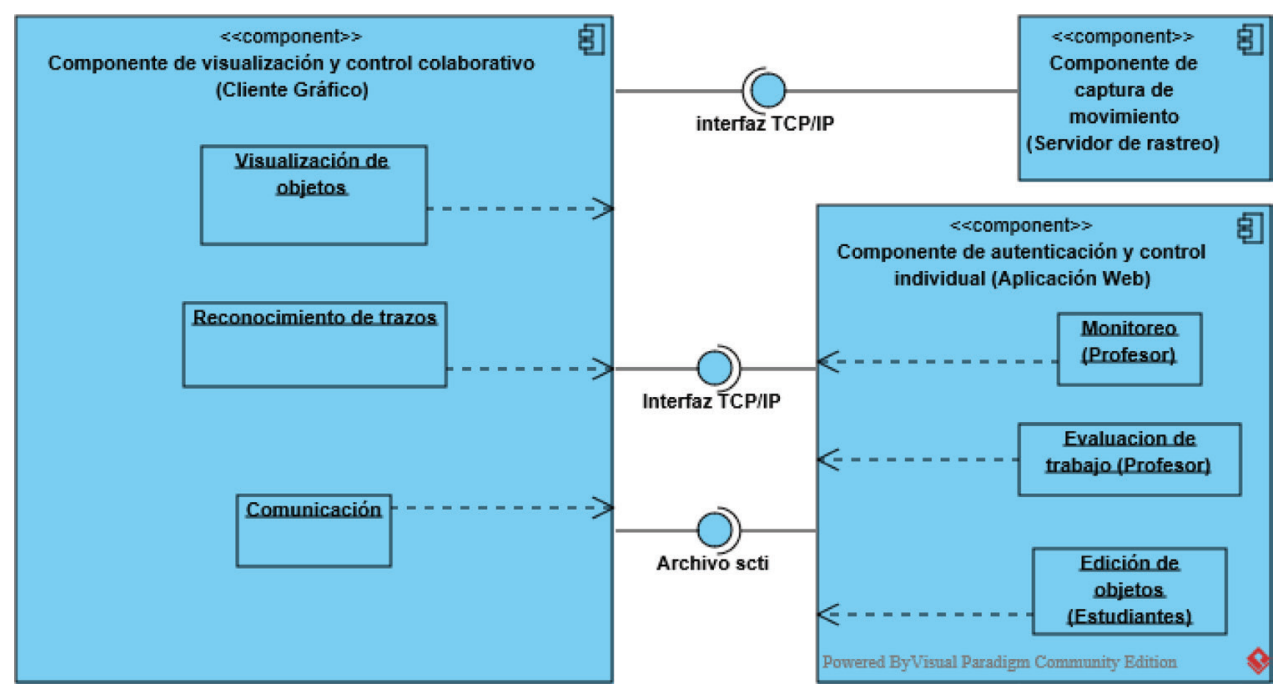

Figura 7 - Diagrama de componentes del sistema 


\section{Resultados experimentales}

Una vez que implementado, se realizó una prueba de funcionalidad para medir el grado de cumplimiento de los requerimientos definidos. Estas pruebas tuvieron un enfoque de caja negra. Los casos de prueba diseñados están definidos en un documento que cumple con el estándar IEEE 829-1998. También se realizó una prueba de usabilidad con el fin de validar el sistema. Esta prueba permitió medir tres aspectos de usabilidad: facilidad de uso, satisfacción del usuario y utilidad del sistema. En este sentido, se seleccionó a 10 profesores del área de ingeniería en ciencias computacionales que tienen relación con la enseñanza y/o evaluación del modelamiento de bases de datos. También, participaron 22 estudiantes del curso de bases de datos I. Los experimentos se organizaron en dos grupos, el de profesores y estudiantes.

Experimento 1: los profesores participaron en un pre-post-test, se utilizó un cuestionario donde se les preguntó acerca de su percepción acerca de sus experiencias en trabajos grupales en relación a las siguientes variables: Equidad de carga de trabajo; facilidad de asignar una calificación individual y grupal. Las variables fueron medidas utilizando la escala de Likert. Posteriormente, se condujo una sesión de trabajo que consistió en la participación de los profesores en el monitoreo y evaluación de una sesión de trabajo colaborativo utilizando la solución implementada. Los profesores a través de la interfaz Web de la solución pudieron monitorear y evaluar las sesiones de trabajo. Una vez finalizada la experimentación, se realizó una segunda observación en la que se volvió a medir las mismas variables.

Experimento 2: los estudiantes participaron en dos experimentos con un esquema pre-post-test, con 7 semanas de diferencia; fueron asignados a grupos de trabajo en los que participaron 3 a 4 estudiantes escogidos al azar. Los estudiantes asignados al grupo experimental participaron utilizando la superficie colaborativa. Los estudiantes en el grupo de control participaron utilizando herramientas tradicionales, las cuales fueron: marcadores de colores, papelógrafo y stikers; o la herramienta Web LucidChart. Se utilizó un cuestionario donde se les preguntó sobre su percepción acerca de las siguientes variables: equidad de carga de trabajo, capacidad de las herramientas que para reflejar su aporte real; y la conformidad con sus calificaciones individuales y grupales. De igual forma, las variables fueron medidas utilizando la escala de Likert.

Las pruebas de funcionalidad realizada a los 3 componentes del sistema que representa la superficie colaborativa, fueron cumplidas con éxito en todos los casos.

La tabla 1 resume los resultados obtenidos en la experimentación con los profesores en donde se muestran las estadísticas descriptivas con los correspondientes valores de Z y P. Se puede observar que, una vez que se utiliza el sistema propuesto, los profesores respondieron que la facilidad de asignar calificación individual y grupal fue mayor. De la misma manera, su percepción de equidad se reporta mayor en el post-test que el pre-test. 


\begin{tabular}{lllll}
\hline \multirow{2}{*}{ Variables } & Pre-Test & Post-Test & & p \\
\cline { 2 - 3 } & Mediana & Mediana & & p \\
\hline $\begin{array}{l}\text { Facilidad de asignar una } \\
\text { calificación individual }\end{array}$ & 2 & 5 & $-2,859$ & 0.004 \\
\hline $\begin{array}{l}\text { Facilidad de asignar una } \\
\text { calificación grupal }\end{array}$ & 4 & 5 & $-2,333$ & 0.020 \\
\hline $\begin{array}{l}\text { Equidad de carga de } \\
\text { trabajo }\end{array}$ & 2 & 4 & $-2,372$ & 0.018 \\
\hline
\end{tabular}

Tabla 1 - Estadísticas descriptivas y pruebas de la percepción de profesores

Se evaluó la usabilidad a través de 3 conceptos distintos con los estudiantes. Para la facilidad de uso del sistema, se obtuvo que el 91\% de los estudiantes calificaron como fácil o muy fácil la utilización de la mesa colaborativa. Mientras que al 9\% opinaron que no era ni fácil ni difícil. En cuanto a la satisfacción del sistema, se obtuvo que el 70\% de los estudiantes opinaron que su experiencia fue satisfactoria. El 30\% restante opinó que su experiencia fue muy satisfactoria. Y por último, para la utilidad del sistema, se obtuvo que el 90\% de los encuestados respondieron que la herramienta era útil o muy útil. Un 10\% mostró una opinión intermedia. En la tabla 2 se muestran los resultados obtenidos, se puede observar que los resultados del grupo experimental en comparación del pre y post test son significativos, en el caso del grupo de control no presenta diferencias significativas.

\begin{tabular}{|c|c|c|c|c|c|}
\hline \multirow{2}{*}{ Variables } & \multirow{2}{*}{ Grupo } & Pre-Test & Post-Test & \multirow{2}{*}{$\mathbf{Z}$} & \multirow{2}{*}{$\mathbf{p}$} \\
\hline & & Mediana & Mediana & & \\
\hline \multirow{2}{*}{$\begin{array}{l}\text { Equidad de carga de } \\
\text { trabajo }\end{array}$} & Exp. & 3 & 5 & -2.54 & 0.011 \\
\hline & Ctrl. & 4 & 4 & -0.63 & 0.527 \\
\hline \multirow{2}{*}{$\begin{array}{l}\text { Capacidad } \\
\text { herramientas para } \\
\text { reflejar el aporte real }\end{array}$} & Exp. & 4 & 5 & -2.97 & 0.003 \\
\hline & Ctrl. & 4 & 4 & -1.19 & 0.234 \\
\hline \multirow{2}{*}{$\begin{array}{l}\text { Conformidad con } \\
\text { calificación individual }\end{array}$} & Exp. & 4 & 5 & -0.44 & 0.66 \\
\hline & Ctrl. & 4 & 5 & -2.13 & 0.03 \\
\hline \multirow{2}{*}{$\begin{array}{l}\text { Conformidad con } \\
\text { calificación grupal }\end{array}$} & Exp. & 4 & 4.5 & -1.67 & 0.09 \\
\hline & Ctrl. & 4 & 4 & -1.89 & 0.058 \\
\hline
\end{tabular}

Tabla 2 - Estadísticas descriptivas y pruebas de la percepción de los estudiantes

\section{Conclusiones y trabajos futuros}

Este artículo tiene como objetivo describir la implementación de un sistema multi-táctil que promueve y facilita la evaluación de los trabajos colaborativos en el aula. Como se pudo evidenciar en la literatura, las actividades de diseño de colaboración son difíciles de evaluar debido al gran esfuerzo que el profesor debe poner al observar cada estudiante en el desempeño de actividades de colaboración. Se ha demostrado que el sistema propuesto es eficaz y útil para el seguimiento de las contribuciones individuales de los 
estudiantes que están involucrados en el trabajo colaborativo, en el caso particular del experimento, en el diseño de bases de datos. Los estudiantes percibieron que con el uso de este tipo de sistemas es más significativo el aprendizaje comparado con el enfoque tradicional (utilizando, por ejemplo, lápiz, papel y pizarrón).

La mayoría de los estudiantes expresaron que no era difícil de llevar a cabo acciones comunes en el sistema, lo que significa que el diseño propuesto se ajusta a las necesidades de los usuarios. Aunque, la contribución de un estudiante se mide cuantitativamente, la calidad de la contribución debe añadirse a la contribución con el fin de tener una medida más fiable y realista. La calidad puede ser medida mediante la realización de un análisis más profundo de las intervenciones del estudiante mientras se hace el diseño colaborativo; por ejemplo se pudiera realizar un análisis multimodal de voz, los gestos y las acciones de entrada al sistema que permitan evaluar objetivamente este aspecto. Sería interesante poder utilizar el sistema dentro de actividades de diseño que están involucradas en el desarrollo de software. Por ejemplo, el desarrollo de diagramas de UML, diagramas de flujo, diagramas BPMN, entre otros. Además, en otras actividades de diseño de ingeniería, tales como el diseño mecánico o industrial.

Como trabajo futuro, se espera el desarrollo del soporte de la captura multimodal de los gestos y el habla de los estudiantes, con el fin de mejorar la evaluación del diseño colaborativo. Por otra parte, la implementación de hardware también se puede mejorar cambiando el enfoque de seguimiento de trazos a través del sistema Optitrack, a un enfoque basado en visión por ordenador.

\section{Referencias}

AlAgha, I., Hatch, A., Ma, L., Burd, L. (2010). Towards a teacher-centric approach for multi-touch surfaces in classrooms, in ACM International Conference on Interactive Tabletops and Surfaces.

Buxton, W., Fitzmaurice, G.W., Balakrishnan, R., Kurtenbach, G. (2000). Large displays in automotive design. IEEE Computer Graphics and Applications 20(4) 68-75.

Chory-Assad, R. M. (2002). Classroom justice: Perceptions of fairness as a predictor of student motivation, learning, and aggression, Commun. Q., vol. 50, no. 1, pp. 58-77.

Cutler, L.D., Fröhlich, B., Hanrahan, P. (1997). Two-handed direct manipulation on the responsive workbench. In Proceedings of the Symposium on Interactive 3D Graphics, ACM Press.

Kaplan (2014). Graduate recruitment report: Employer perspectives. Technical report.

Dillenbourg, P., Evans, M. (2011). Interactive tabletops in education. International Journal of Computer-Supported Collaborative Learning 6(4), 491-514.

Frisch, M., Heydekorn, J., Dachselt, R. (2010). Diagram editing on interactive displays using multi-touch and pen gestures. In 6th International Conference on Diagrams, Volume 6170 of Lecture Notes in Computer Science, Springer Berlin Heidelberg, 182-196. 
Gasca-Hurtado, G., Peña, A., Gómez-Álvarez, C., Plascencia-Osuna, O., CalvoManzano, J. (2015). Realidad virtual como buena práctica para trabajo en equipo con estudiantes de ingeniería. Revista Ibérica de Sistemas y Tecnologías de la Información. Ed. 16, 76-91.

Hayes, J. H., Lethbridge, T. C., Port, D. (2003). Evaluating individual contribution toward group software engineering projects. In Proceedings 25th International Conference on Software Engineering, 622 - 627.

Higgins, S., Mercier, E., Burd, E., Hatch, A. (2011). Multi-touch tables and the relationship with collaborative classroom pedagogies: A synthetic review. International Journal of Computer-Supported Collaborative Learning 6(4), 515-538.

Jones, A., Moulin, C., Barthes, J., Lenne, D., Kendira, A., Gidel, T. (2012). Personal assistant agents and multi-agent middleware for cscw. In IEEE 16th International Conference on Computer Supported Cooperative Work in Design, 72-79.

Martínez, R., Collins, A., Kay, J., Yacef, K. (2011). Who did what? who said that?: Collaid: An environment for capturing traces of collaborative learning at the tabletop. In Proceedings of the ACM International Conference on Interactive Tabletops and Surfaces. New York, NY, USA, 172-181.

Martinez, R., Kay, J., Yacef, K. (2011). Visualisations for longitudinal participation, contribution and progress of a collaborative task at the tabletop, Int. Conf. Comput. Support. Collab. Learn.

Martínez Maldonado, R., Kay, J., Yacef, K., Schwendimann, B. (2012). An interactive teacher's dashboard for monitoring groups in a multi-tabletop learning environment. In Intelligent Tutoring Systems. Volume 7315 of Lecture Notes in Computer Science. Springer Berlin Heidelberg 482-492.

Martinez Maldonado, R., Dimitriadis, Y., Kay, J., Yacef, K., Edbauer, M.-T. (2012). Orchestrating a multi-tabletop classroom, in Proceedings of the 2012 ACM international conference on Interactive tabletops and surfaces.

Mercier, E.M., Higgins, S.E. (2013). Collaborative learning with multi-touch technology: Developing adaptive expertise. Learning and Instruction 25, 13-23.

National Association of Colleges and Employers (2013). The skills and qualities employers want in their class of 2013 recruits. Technical report.

Paulson, B., Hammond, T. (2008). PaleoSketch: Accurate Primitive Sketch Recognition and Beautification. Proceedings of the 13th International Conference on Intelligent User Interfaces, ACM, 1-10.

Rick, J., Marshall, P., Yuill, N.: Beyond one-size-fits-all (2011). How interactive tabletops support collaborative learning. In: Proceedings of the 10th International Conference on Interaction Design and Children. New York, NY, USA, ACM, 109-117.

Shen, C., Shen, C., Ryall, K., Ryall, K., Forlines, C., Forlines, C., Esenther, A., Esenther, A. (2006). Informing the Design of Direct- Touch Tabletops, IEEE Computer Graphics and Applications, volume 26, 36-46. 
Sinmai, K., Andras, P. (2014). Mapping on Surfaces: Supporting Collaborative Work Using Interactive Tabletop, in Collaboration and Technology SE - 29, vol. 8658, Springer International Publishing, 319-334.

Stahl, O., Wallberg, A., Söderberg, J., Humble, J., Fahlén, L.E., Bullock, A., Lundberg, J. (2002). Information exploration using the pond. In Proceedings of the 4th International Conference on Collaborative Virtual Environments. CVE '02, New York, NY, USA, ACM, 72-79.

Tyng, K.S., Zaman, H.B., Ahmad, A. (2011). Visual application in multi-touch tabletop for mathematics learning: A preliminary study. In: Visual Informatics: Sustaining Research and Innovations. Volume 7066 of Lecture Notes in Computer Science, Springer Berlin Heidelberg, 319-328.

Vázquez-Reyes, S., Villa-Cisneros, J., Anaya-Villagrana, J., Barrera-Ambriz, F., LeónSigg, M., Castañeda-Ramírez, C. (2015). Mapeos proyectivos: la base para el funcionamiento del pizarrón interactivo WiimoteWhiteboard. Revista Ibérica de Sistemas y Tecnologías de la Información. Ed. E3, 85-92.

Wellner, P. (1993). Interacting with paper on the digitaldesk. Commun. ACM 36(7) 87-96.

Wobbrock, J. O., Morris, M. R., Wilson, A. D. (2009). User-defined gestures for surface computing, Proc. 27th Int. Conf. Hum. factors Comput. Syst., 1083-1092.

Xambó, A., Hornecker, E., Marshall, P., Jordà, S., Dobbyn, C., Laney, R. (2013). Let's jam the reactable: Peer learning during musical improvisation with a tabletop tangible interface. ACM Trans. Comput.-Hum. Interact. 20(6), 36:1-36:34. 